\title{
THE FEULGEN NUCLEAL REACTION
}

Acid Degradation of Sperm Deoxyribonucleic Acid

W $\mathrm{E}$ have been seeking methods for preparing deoxyribonucleotides and nucleosides by chemical degradation of sperm deoxyribonucleic acid under acid conditions, and thought it of interest to test for liberated aldehydo groups on the various products. The results have proved of some interest in regard to the views of various workers on the interpretation of results of the Feulgen reaction ${ }^{1}$ and more particularly in the light of views described by one of us with another colleague in the following communication.

sperm deoxyribonucleic acid, prepared in typically hbrous form from soft herring roe by the elegant method of Mirsky and Pollister ${ }^{2}$, can be freed from all but traces of protein by chloroform/butanol treatment and appears to be combined with protein in sperm by electrovalent linkages. Such material slowly becomes Feulgen-positive on being steeped for two to three hours in Schiff's reagent at $p H$ 3. This deoxyribonucleic acid can be irreversibly depolymerized by dialysis of a solution of its sodium salt against tap water for several days, or more rapidly by autolysis $(p \mathrm{H} 3)$ at $100^{\circ}$ for 3 minutes. The product, prepared by the dialysis method, when precipitated by acid ethanol $(p \mathrm{H} 3 \cdot 5)$, loses all semblance of its fibrous nature and can be obtained as a white powder of almost crystalline appearance. It is insoluble at an acid $p \mathbf{H}$ and is converted into an insoluble gum on contact with water. On treatment with Schiff's reagent, the gum-like product instantly gives a strongly positive Feulgen-staining reaction which reaches its maximum intensity in about three hours. The dye so formed is completely non-soluble and non-diffusible, the reagent above the dyed nucleic acid remaining clear for more than thirty hours (cf. Stedman $\left.{ }^{3}\right)$.

The most significant finding is that this partly degraded product having a slightly higher nitrogen content than the original $(\mathrm{N} / \mathrm{P}=11 / 3)$ still retained almost completely its content of purine bases, so we have shown, in contrast to Feulgen's original ideas, that it is unnecessary to remove all purine bases to obtain Feulgen-positive material. We believe that polymeric linkages, by which the maximum macromolecular state (such as is possessed by material isolated in the fibrous form) of native deoxyribonucleic acid is maintained, involve highly labile sugar aldehydo linkages, and these can readily be broken by acid or depolymerase enzymes. For more profound chemical degradation under acid conditions we have used sperm deoxyribonucleic acid provided by the Glaxo Laboratories, Ltd., and have treated it initially with 1.5 per cent methanolic hydrogen chloride at room temperature. We anticipated that such a method would protect any sugar aldehydo groups by acetal or glycoside formation, would not convert deoxyribose into $\omega$-hydroxy lævulinic aldehyde and would not remove organically bound phosphate residues.

We have obtained clear evidence that the first components to be liberated are, indeed, the purinecontaining fractions, which pass into the methanolic hydrogen chloride solution, in which they can be estimated by ultra-violet absorption methods, and then isolated in crystalline form or as the picrates. The methanol-insoluble residue remaining after the bulk of the purine bases have been removed corresponds in properties to a methyl derivative $(\mathrm{N}, 7-8$; OMe 6-7 per cent) of the well-known thymic acid4. It is soluble in water, $[\alpha]_{D}+30^{\circ}$, and after mild acid treatment $(p H 3)$ to remove methoxyl groups, or standing in Feulgen's reagent, it gives an intense 'Feulgenpositive' stain with Schiff's reagent. The dye formed is completely soluble in water, and if diffused into protein material is strongly adsorbed by the protein as in Stedman's experiments ${ }^{3}$. From the modified thymic acid, by increasing step-wise the acid concentration in the methanolic hydrogen chloride treatment up to $p \mathrm{H} 3 \cdot 6$, we have obtained a variety of soluble and insoluble fractions which are difficult to characterize precisely.

Using elementary analysis for carbon, hydrogen, nitrogen and phosphorus, Feulgen and Disches estimations, ultra-violet absorption and the usual methods for estimating purine bases, we have obtained evidence of the presence of guanine, adenine, 'sperm guanosine', 'sperm adenylic' acid and deoxyribose phosphates. There invariably remained a residue completely insoluble in methanolic hydrogen chloride consisting apparently almost completely of the more stable 'sperm thymylic' acid, from which crystalline thymine in good yield can readily be obtained by sublimation methods.

We think it highly improbable from the proportion of the nucleotides so far estimated that sperm deoxyribonucleic acid contains a simple tetranucleotide repeating unit, and consider that Levene's ${ }^{6}$ formula will need to be revised to apply to this deoxyribonucleic acid. From the ease of liberation of sugar aldehydo groups, there would appear to be no reason to doubt that the Feulgen reaction in the hands of eytologists does locate deoxyribonucleic acid at the precise site of its occurrence. On the other hand, the varying intensities of the Feulgen colour, as noted particularly by Bauer ${ }^{7}$ and Hillary ${ }^{8}$ (see also Stefano ${ }^{9}$ ), can readily be accounted for by the high lability under acid conditions of deoxyribonucleic acid and more particularly of its deoxyribofuranose component (see following communication).

We thank the local committee of the British Empire Cancer Campaign for a grant in aid of this work.

\section{Chong-EU LI}

M. STACEY

${ }^{1}$ Feulgen, R., and Rosenheck, H., Z. physiol. Chem., 14, 405 (1924).

2 Mirsky, A. E., and Pollister, A. W., Proc. U.S. Nat. Acad. Sci., 28, 344 (1942).

${ }^{3}$ Stedman, E., and Stedman, E., Symposia Soc. Exp. Biol., 1, 232 (1947).

Feulgen, R., and Voit, K., Z. physiol. Chem., 135, 249 (1924).

s Dische, Z., Microchemie, 8, 4 (1930).

- Tipson, R. S., “Adv. in Carb. Chem.", 1, 243 (1945).

'Bauer, H., Z. Zellforsch. u. mikroscop. Anat., 15, 225 (1932).

Hillary, B. B., Bot. Gaz., 101, 276 (1939).

${ }^{\circ}$ di Stefano, H. S., Proc. U.S. Nat. Acad. Sci., 34, 75 (1948).

\section{Mechanism of the Feulgen Nucleal Reaction}

In a previous paper ${ }^{1}$ from these laboratories, it was show that the mechanism for the Dische ${ }^{2}$ diphenylgmine reaction for deoxyribonucleic acid depends upon the conversion by acid treatment of the depxyribose component into $w$-hydroxy lævulinic aldehyde, which then coupled with diphenylamine to give a blue dye with a characteristic absorption band. Extension of this work with Dr. Ethel Teece has led 
to the isolation in 10 per cent yield of an amorphous blue complex possessing the characteristic band.

The view was expressed ${ }^{1}$ that, under the acid conditions of the Feulgen ${ }^{3}$ reaction, the possible formation of this aldehyde presumably accounted also for the reaction with the Feulgen reagent, though it was noted that the colours of Schiff reagentaldehyde reactions were variable. It was pointed out by Danielli" that an aldehyde with such a small molecular weight would readily be washed out of tissues under the correct method of carrying out Feulgen's test, and that some other mechanism must account for the stain. On the other hand, under the conditions of test, where unduly strong acid conditions obtain, such an aldehyde could form a diffusible complex with Schiff's reagent and could account for the observations which in our opinion have misled Stedman in some of his views on chromosome structure. It was obvious, particularly in view of the controversies regarding the Stedmans' claims ${ }^{5}$ to ascertain the true mechanism of the Feulgen reaction. It was clear that for this purpose further knowledge on deoxy sugars, especially in regard to their acid lability and to the relationship between their aldehydo forms and ring structures, was necessary.

Accordingly, we have devised new syntheses for 2 deoxyribose and have synthesized a number of other 2-deoxy sugars with both pyranose and furanose derivatives thereof. In addition, we have synthesized a variety of 2 -deoxy glycosides. We have carried out the quantitative Schiff's test using the sensitive reagent described by Tobie $^{6}$, under a variety of rigidly controlled conditions on both pyranose and furanose derivatives of the ordinary sugars and of their 2-deoxy analogues. It was possible to demonstrate that under identical conditions 2deoxy sugar derivatives con-

tain a significantly higher proportion of the aldehydo form than do the normal sugars, whereas derivatives of the 2-deoxy furanose sugars contain an appreciably higher proportion of the aldehydo form than is contained by the corresponding derivatives of 2-deoxy pyranose sugars. These differences were quite striking in the galactose and glucose series, but less so in the ribose series, in which we have so far not yet obtained crystalline 2-deoxy ribofuranose derivatives. With the latter it will probably need the 2-deoxy ribofuranose phosphates, now in course of preparation, to get the maximum intensity of Schiff's colour. In examining the glycosides of some 2-deoxy sugars, we find that under acid conditions the glycosidic groups of the furanose types are quite remarkably labile.

Now turning to the reaction between aldehydes and Schiff's reagent, we have the view of Wieland and Scheuing?, which seems reasonable, that the aldehyde forms an actual complex with pararosaniline decolorized by sulphur dioxide. Later workers express similar ideas. In view of this work, and also accepting the assumption, for which there is some evidence, albeit slight, in the literature that the deoxy sugar component presumably occurs naturally in deoxyribonucleic acid in the furanose form, we consider that the mechanism of the Feulgen reaction can now be explained as follows.

Under acid conditions, those sugar linkages engaged in polymeric bonding first become broken, and this reaction is followed by the rupture of the linkages attached glycosidically to purine bases. The 2-deoxyribose components thus revealed are still attached through phosphate linkages at $\mathrm{C}_{3}$ and $\mathrm{C}_{5}$ in the main chain of the nucleic acid and consequently are firmly held in the furanose form, which immediately is transformed into a significant proportion of the aldehydo form. The Schiff's reagent then reacts with the revealed aldehydo groups and becomes attached in the manner postulated by Wieland and Scheuing, giving an insoluble coloured macro-molecule of the type: [Nucleic acid-aldehydo groups]-pararosaniline- $\mathrm{SO}_{2}$ dye.

From the work with $\mathrm{Dr}$. $\mathrm{Li}$ outlined above, it would appear to need only a few of the sugar aldehyde residues to be liberated in order to give definite Feulgen-stained material, and it is clear that, in such a complicated molecule, under optimum conditions a maximum amount of the dye can be formed. It is even more apparent how readily varying intensities up to the maximum can arise owing to the lability of sugar aldehyde attachments and to the instability of the 2-deoxyfuranose sugar component itself. Similarly, losses in intensity by over-hydrolysis can now be explained. We believe that in sperm deoxyribonucleic acid, $\mathrm{C}_{1}$ of the deoxy sugar is engaged in two distinct types of linkages, $(a)$ a form of polymeric linkage, and $(b)$ a glycofuranosidic attachment to the purine (and possibly to the pyrimidine) bases, and that both types are acid-labile. The polymeric type could be no more than some enolic form giving hydrogen bonding which, for example, could be achieved as follows :

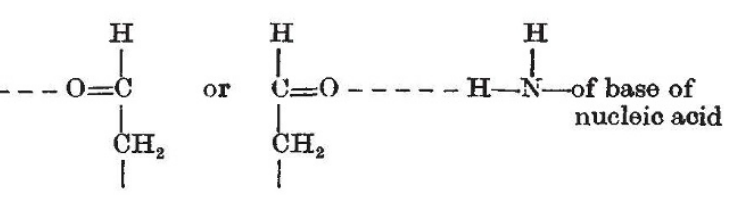

Alternatively, it could involve highly labile 'glyconidic' phosphate residues.

Two important conclusions appeared to come from our work: (1) the explanation of the purine-sugar attachments as being of the 2-deoxy glycofuranosidic type; and (2) if properly carried out, the Feulgen reaction can locate a deoxyribonucleic acid at the site where it occurs. To prove (2), protein-free sperm deoxyribonucleic acid was treated in a citrate buffer at $p \mathrm{H} 3$ at $100^{\circ} \mathrm{C}$. for 2.75 minutes (Widström modification of the Feulgen reaction) and then cooled by plunging into ice-water. The liberated purines were estimated in the solution by ultra-violet absorption spectra measurements. The polymeric material present was collected by adding ethanol and physiological saline', and was washed exhaustively with dilute saline and water. On addition of Schiff's reagent the solid instantaneously developed an intense Feulgen-positive stain, the solution above it remaining completely colourless even after twenty-four hours standing. This illustrated that no diffusion could have occurred such as is postulated by the Stedmans ${ }^{10}$. Moreover, control experiments showed that certain simple aldehydes could be washed out of proteins, nucleic acids and nucleoproteins which had been steeped in these aldehydes; consequently an aldehyde of small molecular weight could not be responsible for any diffusible stain which could be absorbed on to the protein (cf. ref. 10).

We would emphasize that we believe that, in the hands of the cytologist, the Feulgen reaction 
does locate the precise site of deoxyribonucleic acid.

We thank the Board of the British Rubber Producers' Research Association for a grant.

W. G. OvEREND

M. STACEY

Chemistry Department,

University of Birmingham. Sept. 22.

${ }^{1}$ Deriaz, R. E., Stacey, M., Teece, E. G., and Wiggins, L. F., Nature, 157, 740 (1946)

2 Dische, Z., Microchemie, 8, 4 (1930).

${ }^{3}$ Feulgen, R., and Rosenbeck, H., Z. physiol. Chem., 135, 203 (1924).

- Danielli, J. F., Symposia Soc. Exp. Biol., 1, 101 (1947).

s Stedman, E., and Stedman, E., Biochem. J., 43, xxiii (1948).

'Tobie, W. C., Indust. Eng. Chem., 14 (5), 405 (1942).

'Wieland, H., and Scheuing, G., Ber., 54, 2527 (1921); see also Rumpf, P., Ann. Chim., 3, 327 (1935).

${ }^{8}$ Widström, G., Biochem. Z., 199, 298 (1928).

${ }^{9}$ Gulland, J. M., and Jordan, D. O., J. Chem. Soc., 1129 (1947).

10 Stedman, E., and Stedman, E., Symp. Soc. Exp. Biol., 1, 232 (1947), and previous publications.

\section{PROPERTIES OF THE $\propto-P A R T I C L E$ SPARK COUNTER}

\section{By R. D. CONNOR}

Department of Natural Philosophy, University of Edinburgh

T $T$ is the object of this communication to give a 1 preliminary account of certain investigations of the Rosenblum-type spark counter for $\alpha$-particles. In the course of his work it has been shown that the original wire-plate arrangement ${ }^{1}$ is a particular case of a more general type of counter in which the anode is a fine wire and the cathode may be convex, planar or concove with respect to the anode, all these countes operating in air at atmospheric pressure, with electrode separations of the order of $1 \mathrm{~mm}$.

The anode should be a wire of about $0.05 \mathrm{~mm}$. diameter and, since it is important that this wire be kept taut, it should be of a material capable of withstanding considerable tension. Tungsten was chosen as the most suitable anode material on this account. Several cathode materials have been tested, and copper and brass have been found to be as good as any others, the cathode surface being polished to remove irregularities. If a wire cathode is used to give a convex cathode surface, the diameter of the cathode should be several times that of the anode wire, the axes of the two electrodes being parallel. U sing a hollow cylinder to give a concave cathode, a slit or window should be provided in the cylinder to permit entry of the $\alpha$ particles into the sensitive volume.
With the cathode connected to a negative hightension supply, stabilized but variable up to $4 \mathrm{kV}$., and a resistance-capacity potentiometer connected from anode to earth, such arrangements have been found to be simple and convenient $\alpha$-counters, giving visible and audible sparks when a source is brought within $\alpha$-range of the counter. The potentiometer serves to quench the spark discharge; also, by tapping off a small fraction of the impedance of the potentiometer, a pulse of suitable size can be obtained for the operation of a scaler or cathode ray oscillograph. In this connexion it has been shown that the pulse developed across the total quench impedance is approximately equal to the voltage appearing across the electrode gap prior to discharge. The leading edge of the pulse is sharp, the time of rise being estimated as less than $10^{-7}$ sec. The recovery-time is largely determined by the time-constant of the quench circuit, and values of the order of $50 \mathrm{usec}$. have been found convenient. It has not been found possible to achieve stable operation when the timeconstant is less than about 20 usec.

For the counter to spark, the electron avalanche reaching the anode must exceed a critical size. The probability of sparking will therefore be a function of the gas pressure, the geometrical arrangement and applied voltage which produces the field in the electrode gap, and the specific ionization of the $\alpha$-particle in the sensitive region of the counter. It is found experimentally, over a wide variety of conditions, that the counting efficiency varies with the residual range of the $\alpha$-particle, being a maximum when the residual range is small. $\beta$-Rays are not counted, the avalanches they create being insufficient to produce a spark; also, in the absence of an $\alpha$-emitting contamination, the background is zero.

In all its forms the spark counter is 'directional', having greatest sensitivity for $\alpha$-particles arriving normally to the anode wire and very small sensitivity for particles entering parallel to the electrodes. With

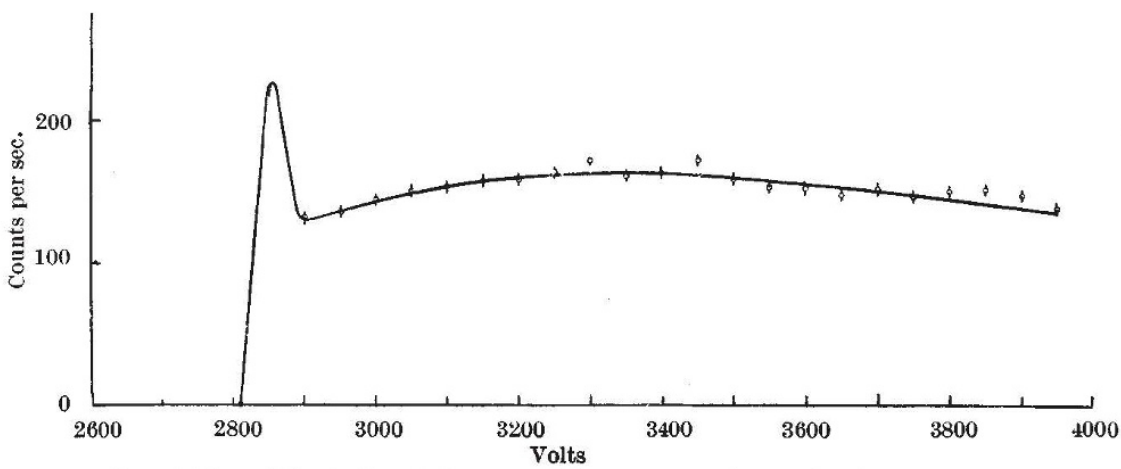

Fig. 1. 'Broadside position.' Gas, air (pressure $76.7 \mathrm{~cm}$. mercury). Anode wire, tungsten $0.05 \mathrm{~mm}$. diameter. Cathode wire, copper $0.9 \mathrm{~mm}$. diameter. Electrode spacing $0.75 \mathrm{~mm}$.

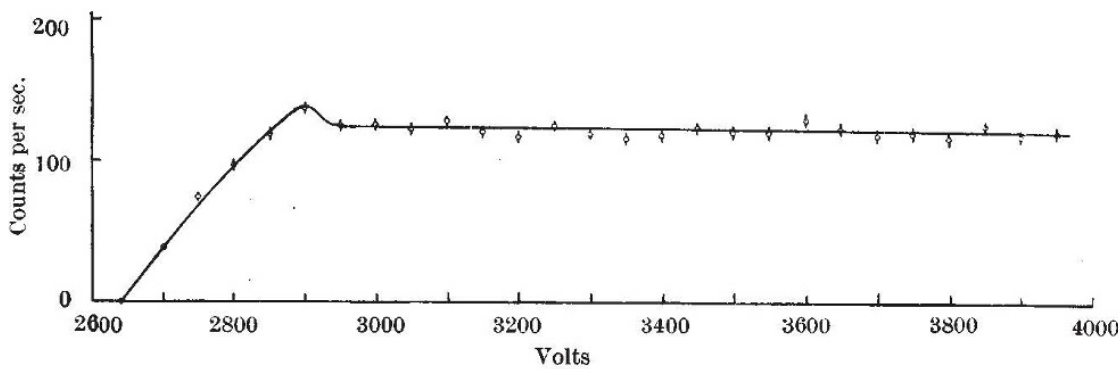

Fig. 2. 'End-on position.' Same counter as in Fir 1 